\title{
A straightforward estimation of the maximum sunspot number for cycle 23
}

\author{
B. Mendoza and J. Ramírez \\ Insituto de Geofisica UNAM, Ciudad Universitaria, 04510 México D.F., MEXICO \\ E-mail: blanca@tonatiuh.igeofcu.unam.mx
}

Received: 5 June 1998 / Revised: 8 October 1998 / Accepted: 3 November 1998

\begin{abstract}
Using the annual number of geomagnetically quiet days $(a a<20 \gamma)$ for the year after the solar minimum, this precursor method predicts that the maximum sunspot number for cycle 23 will be $140 \pm 32$, indicating that cycle 23 will be similar to cycles 21 and 22 .
\end{abstract}

Key words Solar physics - astrophysics and astronomy (magnetic fields; general)

\section{Introduction}

Estimating the strength of solar cycle 23 serves various purposes. It has a practical interest in helping to prevent damaging effects of solar phenomena on integral parts of low-orbit space stations and radio communications. It provides information on the physical processes inside the Sun. It allows us to assess further the relationship found between solar irradiance and solar activity (see for instance review by Lean, 1997) which has a very direct influence on weather and climate; in particular the positive correlation found between solar activity, through the amplitude of the cycle, and average Earth's temperature (Friis-Christensen and Lassen, 1991).

The most successful prediction methods are the precursor techniques. They are based on the premise that the solar cycle is composed of two kinds of cycles which co-exist on the Sun for some years (the poloidal and the toroidal cycles). Under this scheme it is possible to find correlations between the amplitude of the next cycle, and phenomena on the Sun or originated from the

Correspondence to: $\mathrm{B}$. Mendoza
Sun during the declining phase or near the minimum of the cycle in progress.

For instance Schatten et al. (1978) suggested that the Sun's polar field strength near solar minimum is closely related to the following solar cycle. Schatten and Pesnell (1993) obtained an index describing the amount of the buoyant solar magnetic field flux near minimum, and predicted a sunspot maximum number for cycle 23 of $170 \pm 25$. More recently Schatten et al. (1996) predicted a lower value of $138 \pm 30$.

Ohl (1966) found that geomagnetic activity during the last years of the solar cycle could be useful for predicting the next cycle. Later Simon and Legrand (1986) determined that this kind of activity originates from streams coming from polar coronal holes. Thompson (1993) assumed that there are two components of geomagnetic activity and that the total number of geomagnetic disturbances during a solar cycle should depend on both, the amplitude of the current cycle as well as the amplitude of the next one. He identified the disturbed days from the $A p$ index with $A p \geq 25$, and used a multiple linear regression analysis finding the total number of geomagnetic disturbances along a solar cycle as a function of the maximum sunspot number of the cycle in progress and the next one. Recently Thompson (1996) predicted a sunspot maximum number for cycle 23 of $\sim 164$. Li (1997) decomposed the smoothed monthly mean geomagnetic $A p$ indices into two components and explored the relation between one of these and the sunspot number; she predicted a maximum sunspot number for cycle 23 of $149.3 \pm 19.9$. Kane (1997) used monthly means, 12-month moving averages and annual averages, and performed correlations between the $a a$ indices at the year of the minimum and sunspot numbers for the year of the maximum and minimum. He found the highest correlation for 12month moving averages between the maximum sunspot number of the following cycle and the $a a$ value for the minimum year of the cycle in progress. For cycle 23 he predicted $170 \pm 13$ with a single variable regression analysis and $177 \pm 21$ for a bivariate analysis. 


\section{The physical model}

It has been established that low velocity winds are associated with the helmet streamers and the interplanetary current sheet (Kojima and Kakinuma, 1990). There is also good association between the $a a$ indices and solar wind velocity (Mayaud, 1973; Svalgaard, 1977). Therefore we should expect that the less geomagnetically perturbed days, the quiet days, are associated with the lowest velocity winds, which flow through the heliosheet.

Simon and Legrand (1987) studying cycles 11 to 21 found that at the peak of the quiet days the heliosheet has its maximum thickness; this occurs at the end of the dipole phase when the solar dipole is weakest, near solar minimum. It has been also shown that the evolution of the poloidal field is in advance by 5 to 6 years on the toroidal field emergence (Simon and Legrand, 1986).

These findings led Simon and Legrand (1987) to propose an inverse relation between the thickness of the heliosheet near sunspot minimum and the sunspot maximum number of the next cycle. As the thickness is directly related to the number of geomagnetically quiet days, this relation can be used to find a correlation between the maximum number of quiet days around the minimum of the current cycle and the sunspot maximum number of the following cycle. Then a weak poloidal field evidenced through a high number of quiet days will generate a weak toroidal field in the next cycle with a low maximum sunspot number and viceversa.

\section{Results and discussion}

Given the inverse relationship between the quiet days around the solar minimum of the cycle in progress and the sunspot maximum number of the following cycle, we obtain a linear relationship using the geomagnetically quiet days, defined as those with $a a<20 \gamma$, through the years 1868 to 1997 , cycles 11 to 22, as reported by the Solar Geophysical Data. The behaviour of the maximum number of quiet days was analysed for each cycle: $8(73 \%)$ out of the 11 solar cycles studied had a maximum number of quiet days $1 \mathrm{y}$ after the solar minimum, 2 cycles $(18 \%)$ during the year of the minimum and 1 cycle $(9 \%) 1$ year before the solar minimum.

Figure 1 shows the linear regression between the annual number of quiet days, aa $<\gamma$, for the year after the minimum of the cycle in progress and the maximum sunspot number of the following cycle $R m$, we used cycles 11 to 22 . The equation of the linear regression is:

$R m=-(0.85 \pm 0.1) a a+(364.44 \pm 34.3)$

with $r=0.92$.

We consider then the linear regressions between the maximum sunspot number of the next cycle, $R m$, and: (a) the annual number of quiet days of that year which has the maximum number of quiet days along the cycle in progress, and (b) the annual number of quiet days for the years before, during and after the solar minimum of

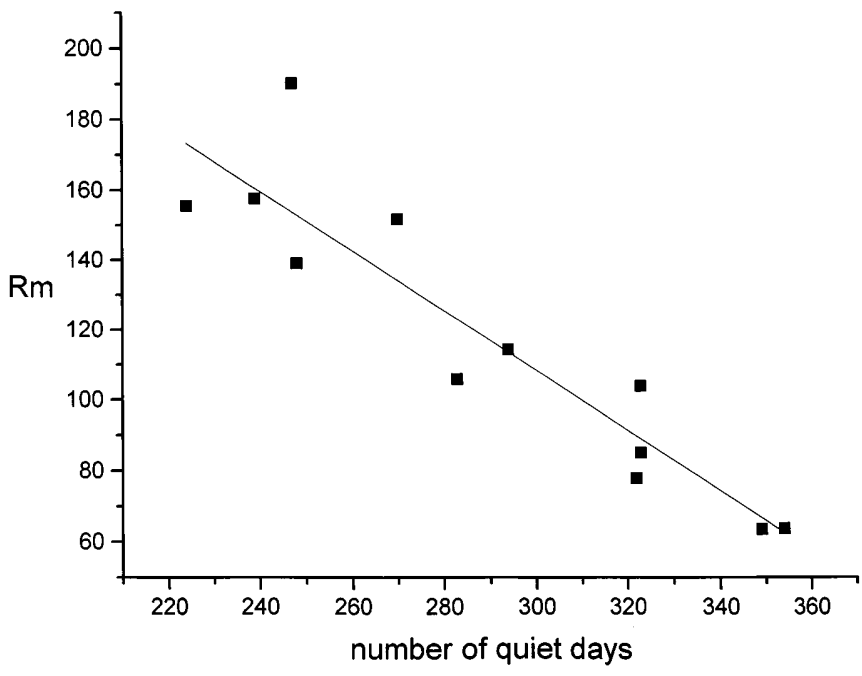

Fig. 1. Linear regression between the annual number of quiet days $(a a<20 \gamma)$ of the year after the minimum of the cycle in progress and the maximum sunspot number of the next cycle $R m: R m=$ $-(0.85 \pm 0.1) a a+(364.44 \pm 34.3)$, with $r=0.92$. Cycles 11 to 22 are used

the current cycle. We found correlations of 0.91 and 0.85 respectively.

The best correlation is the one given by the annual number of quiet days corresponding to the year after the solar minimum. In Table 1 we present the predicted maximum sunspot numbers using Eq. (1) for cycles 11 to 23. Column 1 indicates the number of the cycle, column 2 the predicted maximum sunspot number, column 3 the observed maximum sunspot number and columns 4 and 5 present the lower and upper limits of the predicted maximum sunspot number, the limits were obtained at the $99.9 \%$ confidence level. Taking into account the level of confidence, the predicted values coincide with the observed ones.

The advantage of the method presented is its simplicity. We only need to wait until the year after the solar minimum has passed, calculate the annual number of days with $a a<20 \gamma$ during that year, and apply Eq. (1) in order to obtain the sunspot maximum

Table 1 Observed and predicted maximum sunspot numbers

\begin{tabular}{lrrrr}
\hline Cycle & $R p$ & $R o$ & Lower limit & Upper limit \\
\hline 11 & 153 & 139 & 116 & 190 \\
12 & 63 & 64 & 33 & 92 \\
13 & 89 & 85 & 66 & 112 \\
14 & 67 & 64 & 38 & 95 \\
15 & 89 & 104 & 66 & 112 \\
16 & 90 & 78 & 67 & 113 \\
17 & 114 & 114 & 90 & 137 \\
18 & 134 & 152 & 105 & 164 \\
19 & 154 & 190 & 116 & 191 \\
20 & 123 & 106 & 97 & 149 \\
21 & 173 & 155 & 127 & 220 \\
22 & 161 & 158 & 120 & 201 \\
23 & 140 & & 108 & 172 \\
\hline
\end{tabular}

$R p$, predicted sunspot values using Eq. (1); Ro, observed sunspot value 
number of the next solar cycle. In particular our prediction for cycle 23 using Eq. (1) is $140 \pm 32$.

We now proceed to compare these result with previous estimations. The NOAA Space Environment Center with the support of NASA Office of Space Science has convened the Solar Cycle 23 Project Panel formed by 12 scientists. The conclusion reached by the panel is that it should be expected a smoothed sunspot maximum of $\sim 160$ for cycle 23 . Within this study, the precursor techniques in particular predicted a maximum sunspot number of 160 with 140 at the low end of the range and 180 at the high end of the range (Joselyn et al., 1997). Our prediction is close to the lower end of the range reported. This indicates that cycle 23 will be similar to cycles 21 and 22 .

\section{Conclusion}

Using the annual number of quiet days as given by the $a a$ indices $(<20 \gamma)$ for the year after the solar minimum, we predict that the maximum sunspot number for cycle 23 will be $140 \pm 32$. This indicates that cycle 23 will be similar to cycles 21 and 22 .

Acknowledgement. Topical Editor R. Schwenn thanks R. J. Thompson for his help in evaluating this paper.

\section{References}

Friis-Christensen, E., and K. Lassen, Length of the solar cycle: an indicator of solar activity closely associated with climate, Science, 254, 698, 1991.

Joselyn, J. A., J. B. Anderson, H. Coffey, K. Harvey, D. Hathaway, G. Heckman, E. Hildner, W. Mende, K. Schatten, R. Thompson,
A. W. P. Thomson, and O. R. White, Panel achieves consensus prediction of solar cycle 23, EOS, 78, 205, 1997.

Kane, R. P., A preliminary estimate of the size of the coming solar cycle 23, Geophys. Res. Lett., 24, 1899, 1997.

Kojima, M., and T. Kakinuma, Solar cycle dependence of global distribution of solar wind speed, Space Sci. Rev., 53, 173, 1990.

Lean, J., The Sun's variable radiation and its relevance for Earth, Ann. Rev. Astron. Astrophys., 35, 33, 1997.

Li, Y., Predictions of the features for sunspot cycle 23, Solar Phys., 170, 437, 1997.

Mayaud, P. N., A hundred year series of geomagnetic data, 18681967, IAGA BULL:, 33, Zurich, 251p., 1973.

Ohl, A. I., Forecast of sunspot maximum number of cycle 20. Soln. Danie, 12, 84, 1966.

Schatten, K. H., P. H. Scherrer, L. Svalgaard, and J. M. Wilcox, Using dynamo theory to predict the sunspot number during solar cycle 23, Geophys. Res. Lett., 5, 411, 1978.

Schatten, K. H., and W. D. Pesnell, An early dynamo prediction: cycle $23 \sim 22$, Geophys. Res. Lett., 20, 2275, 1993.

Schatten, K. H., B. J. Myers, and S. Sofia, Solar activity forecast for solar cycle 23. Geophys. Res. Lett., 23, 605, 1996.

Simon, P. A., and J. -P. Legrand, Some solar cycle phenomena related to the geomagnetic activity from 1868 to 1980 II. High velocity wind streams and cyclical behaviour of poloidal field, Astron. Astrophys., 155, 227, 1986.

Simon, P. A., and J. -P. Legrand, Some solar cycle phenomena related to the geomagnetic activity from 1868 to 1980 III. Quietdays, fluctuating activity or the solar equatorial belt as the main origin of the solar wind flowing in the ecliptic plane, Astron. Astrophys., 182, 329, 1987.

Svalgaard, L., Geomagnetic activity independence on solar wind parameters, in Coronal holes and high speed streams, ed. J. B. Zirker, Colorado Ass. Univ. Press, Boulder, 371, 1977.

Thompson, R. J., A technique for predicting the amplitude of the solar cycle, Solar Phys., 148, 383, 1993.

Thompson, R. J., The amplitude of solar cycle 23, Proc. Solar Terrestrial Predictions V, Proceedings of a workshop at Hitachi, Japan, January 1996. 